IFT-UAM/CSIC-09-03

February $20^{\text {th }} 2009$

\title{
Supersymmetric non-Abelian black holes and monopoles in Einstein-Yang-Mills SUGRAS
}

\author{
Mechthild Hübscher, Patrick Meessen, Tomás Ortín and Silvia Vaulà \\ Instituto de Física Teórica UAM/CSIC Facultad de Ciencias C-XVI, \\ C.U. Cantoblanco, E-28049-Madrid, Spain \\ e-mail: \{Mechthild.Huebscher, Patrick.Meessen , Tomas.Ortin , \\ Silvia.Vaula\}@uam.es
}

\begin{abstract}
We discuss the construction of non-Abelian black holes and globally regular monopole solutions to $N=2 d=4$ EYM theories. Special emphasis is put on how the attractor mechanism works for the non-Abelian black holes.
\end{abstract}

\footnotetext{
${ }^{1}$ Contribution to the proceedings of the 4th Workshop RTN "Constituents, Fundamental Forces and Symmetries of the Universe" Varna (Bulgaria) 11-17 September 2008, to be published in Fortschritte der Physik - Progress of Physics
} 
The class of supersymmetric solutions in ungauged $N=2 d=4$ supergravity theories that can give rise to black hole spacetimes is completely characterized [1]: the most general of such solutions is parametrized by an even number of real functions that are harmonic on $\mathbb{R}^{3}$ and to which we shall refer as seed functions. Given these seed functions there exists an algorithm to express all the physical fields in terms of them. Unsurprisingly, not every choice for the seed functions leads to a regular black hole spacetime, but the necessary and sufficient conditions that allow for a static, asymptotically flat, spherically symmetric black hole are known (see e.g. [2]). Apart from the absence of NUT charge and the positivity of the ADM mass, the only remaining condition one has to impose is the existence of a horizon. Having localized the horizon, the near horizon geometry, i.e. the geometry obtained by zooming in on the horizon, is that of an $a D S_{2} \times S^{2}$ geometry and the entropy of the black hole can then be read off easily by using the area law.

The surprising outcome of the above story is that the entropy of the resulting black hole depends only on the electric and magnetic charges of the various Maxwell fields, and not on the asymptotic values of the scalar fields. As befitting uncharged scalar fields, they are constant on the horizon, but their horizon values are also only given in terms of the electric and magnetic charges of the Maxwell fields. This fact goes under the name of attractor mechanism [3] and is fundamental to the successful matching of the entropy calculated in supergravity with the microscopical one in string theory [4].

These results have not been extended so far to black holes with non-Abelian charges. In order to obtain such solutions, the natural setup is gauged $N=2 d=4$ supergravity coupled to non-Abelian vector supermultiplets. For sake of simplicity we shan't introduce hypermultiplets. Whence only isometries of the special-Kähler manifold parametrized by the scalars in the vector multiplets are gauged and we shall ignore possibility of turning on a Fayet-Iliopoulos term. We refer to these models as $N=2 d=4$ Einstein-Yang-Mills (EYM) theories. As in the Abelian case, one can proceed to characterize the supersymmetric solutions in terms of seed functions and select those that generate regular black hole solutions. Unlike the Abelian case where the Bianchi and Maxwell equations imply that the seed functions are harmonic on $\mathbb{R}^{3}$, however, the non-Abelian case allows for less trivial field configurations. We can in fact rephrase the problem in terms of finding among the solutions to the Bogomol'nyi equations for a given Yang-Mills-Higgs system, those that, when embedded into a suitable EYM theory, give rise to regular non-Abelian black holes. As an example we will treat the embedding of unit-charge solutions to the $\mathrm{SO}(3) \mathrm{YMH}$ system into the $\overline{\mathbb{C P}}^{3}$ EYM model.

Remarkably, we find that even in the non-Abelian case some kind of attractor mechanism is at work. In fact, we find well-defined hairy non-Abelian black holes, with hair that is asymptotically invisible and does not contribute to the properties of the horizon. In these hairy solutions the entropy of the black hole depends only on the asymptotic color charges (See e.g. Ref. 8] for more information on the definition of color charge). The scalars on the horizon depend also only on the color charges, but are not constant over it, rather they form a hedgehog configuration; this was to be expected as we are dealing with scalars transforming in the adjoint of the gauge group. The idea that the attrac- 
tor mechanism is expressible in terms of asymptotic charges is, however, not true as will be seen by the construction of a colored black hole: for such black holes, no asymptotic charges exist, yet the entropy of the black hole receives contributions from the unit-charge YMH-configuration.

As mentioned, we shall consider the $\overline{\mathbb{C P}}^{3}$ EYM model, which is based on the special geometry of the $S U(1,3) / U(3)$ coset space. The model features one gravitational multiplet coupled to three vector multiplets. The bosonic field content of this model consists of the metric, 4 gauge fields $A^{\Lambda}(\Lambda=0,1,2,3)$ and 3 complex scalar fields $Z^{i}(i=1,2,3)$. The fermionic field content consists in two gravitini $\psi_{I \mu},(I=1,2)$ and six gaugini $\lambda^{I i}$, two for each vector multiplet, transforming as doublets under the $\mathrm{SU}(2) \mathrm{R}$-symmetry group. We refrain from gauging the $\mathrm{U}(2) \mathrm{R}$-symmetry group and choose an $S O(3)$-gauging, where 3 of the vector fields, $A^{i}$, constitute the gauge fields, leaving $A^{0}$ as an Abelian field; the scalars and the doublets of gaugini transform as a triplet under the $S O(3)$.

The bosonic action for the model is

$$
\int_{4} \sqrt{g}\left[\frac{1}{2} R+\mathcal{G}_{i \bar{j}} \mathfrak{D}_{a} Z^{i} \mathfrak{D}^{a} \bar{Z}^{\bar{j}}-V(Z, \bar{Z})+\operatorname{Im}(\mathcal{N})_{\Lambda \Sigma} F_{a b}^{\Lambda} F^{\Sigma a b}-\operatorname{Re}(\mathcal{N})_{\Lambda \Sigma} F_{a b}^{\Lambda}(\star F)^{\Sigma a b}\right],
$$

and the field strengths and the covariant derivatives are given by

$$
F^{0}=d A^{0}, F^{i}=d A^{i}+\frac{g}{2} \varepsilon_{j k}{ }^{i} A^{j} \wedge A^{k}, \mathfrak{D} Z^{i}=d Z^{i}+g \varepsilon_{j k}{ }^{i} A^{j} Z^{k},
$$

where $g$ is the coupling constant. As the metric $\mathcal{G}$ is Kähler it can be derived from a Kähler potential, $\mathcal{K}$, which for the chosen model reads $e^{-\mathcal{K}}=1-|Z|^{2}$. Observe that the Kähler potential imposes the constraint $0 \leq|Z|^{2} \leq 1$, but it can be shown that a BH solution automatically satisfies this bound [2]. The explicit form of the complex matrix $\mathcal{N}$ and the potential $V$, which by construction is positive semi-definite, can be written down, but as they will not be used we shall refrain from doing it.

In order to find supersymmetric solutions it is convenient to identify first the bosonic field configurations that admit at least one Killing spinor. This is achieved by looking for solutions to the Killing Spinor Equations (KSEs)

$$
\delta_{\epsilon} \psi_{I \mu}=\delta_{\epsilon} \lambda^{I i}=0,
$$

whose explicit form can be found e.g. in Ref. [5]. Afterwards one imposes the equations of motion, thus sorting the supersymmetric solutions out of the supersymmetric configurations. This task is specially simple due to the existence of Killing Spinor Identities (KSIs) relating the off-shell e.o.m.s of supersymmetric configurations. As a consequence one must explicitly solve just a limited number of equations of motion, the others being implied by the KSIs. In the case of interest, that is when the metric admits a timelike Killing vector, it is enough to verify that the Maxwell equations and the Bianchi identities of the vector fields are satisfied.

The static metric admitting a timelike Killing vector can be conveniently written in the form

$$
d s^{2}=K^{-1} d t^{2}-K d x^{m} d x^{m}, \quad m=1,2,3 .
$$


The function $K$ determines the whole metric and is expressed in terms of the seed function according to

$$
K=\langle\mathcal{R}, \mathcal{I}\rangle=\mathcal{I}^{\Lambda} \mathcal{R}_{\Lambda}-\mathcal{I}_{\Lambda} \mathcal{R}^{\Lambda}
$$

where $\mathcal{I} \equiv\left(\mathcal{I}^{\Lambda}, \mathcal{I}_{\Lambda}\right)$ is the symplectic vector of real, seed functions, and $\langle$,$\rangle is, as indicated,$ a symplectic product. The symplectic vector $\mathcal{R} \equiv\left(\mathcal{R}^{\Lambda}, \mathcal{R}_{\Lambda}\right)$ is not independent but is related to $\mathcal{I}$ via the model-dependent stabilization equations. For our chosen model the solution to the stabilization equations read

$$
\mathcal{R}_{\Lambda}=-\frac{1}{2} \eta_{\Lambda \Sigma} \mathcal{I}^{\Sigma} ; \quad \mathcal{R}^{\Lambda}=2 \eta^{\Lambda \Sigma} \mathcal{I}_{\Sigma}
$$

The seed functions are defined in such a way that the scalar fields read

$$
Z^{i}=\frac{\mathcal{R}^{i}+2 i \mathcal{I}^{i}}{\mathcal{R}^{0}+2 i \mathcal{I}^{0}}
$$

while the expression for the field strengths provided by the KSEs is

$$
F^{\Lambda}=-\frac{1}{\sqrt{2}} \mathfrak{D}\left(K \mathcal{R}^{\Lambda} d t\right)-\frac{1}{\sqrt{2}} K \star\left(d t \wedge \mathfrak{D} \mathcal{I}^{\Lambda}\right)
$$

Staticity imposes the following relation among the seed functions

$$
\left\langle\mathcal{I}, \mathfrak{D}_{m} \mathcal{I}\right\rangle=0
$$

From (0.8) we can impose the Bianchi identities of the vector fields, which turns out to be equivalent to

$$
\mathfrak{D}_{m} \mathfrak{D}_{m} \mathcal{I}^{\Lambda}=0 \text {. }
$$

The Maxwell/Yang-Mills equations, on the other hand give rise to

$$
\mathfrak{D}_{m} \mathfrak{D}_{m} \mathcal{I}_{\Lambda}=\frac{g^{2}}{2}\left[\varepsilon_{\Lambda(\Sigma} \varepsilon_{\Delta) \Gamma}{ }^{\Omega} \mathcal{I}^{\Sigma} \mathcal{I}^{\Delta}\right] \mathcal{I}_{\Omega}
$$

where only the $\varepsilon_{i j}{ }^{k}$ are non-vanishing. Note that (0.11) ensures the integrability of the staticity condition (0.9).

Equations (0.10) and (0.11) are in general not easy to solve. The best strategy is to start with a given $A^{\Lambda}$, and try to deduce $\mathcal{I}^{\Lambda}$ by comparing the resulting field strength in Eq. (0.8). Doing this implies that Eq. (0.10) is equivalent to the Bogomol'nyi equation

$$
\mathfrak{D}_{p} \mathcal{I}^{\Lambda}=-\frac{1}{\sqrt{2}} \epsilon_{p m n} F_{m n}^{\Lambda},
$$

where one should observe that the integrability condition for the Bogomol'nyi equation is nothing but Eq. (0.10). Moreover, the $\mathcal{I}^{\Lambda} \mathrm{s}$ that are not charged under the gauge group, e.g. in our case $\mathcal{I}^{0}$, are as before harmonic functions on $\mathbb{R}^{3}$.

In the ungauged case, i.e. when $g=0$, the 6 seed functions in the spherically symmetric case are given by

$$
\mathcal{I}^{\Lambda}=h^{\Lambda}+\frac{p^{\Lambda}}{r}, \mathcal{I}_{\Lambda}=h_{\Lambda}+\frac{q_{\Lambda}}{r},
$$


where $p^{\Lambda}$ and $q_{\Lambda}$ are the magnetic and electric charges of the 4 Maxwell fields. As mentioned above, given the seed functions we can generate the complete expressions of the fields, and in this letter, we shall be interested in static BH-like solutions. Then, staticity imposes the constraint $h_{\Lambda} p^{\Lambda}-h^{\Lambda} q_{\Lambda}=0$ which is equivalent to imposing the vanishing of a possible NUT charge. Here we shall solve this constraint by putting $\mathcal{I}_{\Lambda}=\mathcal{R}^{\Lambda}=0$, so that we will be dealing with purely magnetic solutions only. The supergravity fields can then be expressed in terms of the seed functions a: 2

$$
\vec{Z}=\frac{\overrightarrow{\mathcal{I}}}{\mathcal{I}^{0}}, 2 K=(\mathcal{I})^{2}-\overrightarrow{\mathcal{I}}^{2}
$$

Without loss of generality we can normalize the solution to asymptote to ordinary Minkowski space by putting $\left(h^{0}\right)^{2}=2+\vec{h}^{2}$. The ADM mass can be seen to be $2 M=h^{0} p^{0}-\vec{h} \cdot \vec{p}$ and must be taken to be positive.

As one can see, there is a possible horizon located at $r=0$; the condition for the solution to describe the geometry outside a regular black hole is then equivalent to the statement that the geometry in the limit $r \rightarrow 0$, is that of a well-defined $a D S_{2} \times S^{2}$ space, with the $S^{2}$ being spacelike. In case this is true, the area of the 2 -sphere gives rise to the black hole's entropy. Of course, if we just calculate the limit and identify the would-be area of the 2-sphere without bothering its well-definedness, we find an expression for area/entropy which might be negative. By an abuse of language, then, we say that the condition for the existence of a horizon is equivalent to the positivity of the entropy.

Applying the above reasoning to our Abelian set-up then means that we have a black hole if

$$
2 S_{b h}=\left(p^{0}\right)^{2}-\vec{p}^{2}>0 .
$$

Observe that this entropy, conforming to the attractor mechanism, only depends on the magnetic charges. Furthermore, the expression for the scalars on the horizon reads $\vec{Z}=$ $\vec{p} / p^{0}$, which also fully conforms to the attractor mechanism.

The general class of supersymmetric solutions to $N=2 d=4$ SUGRA with YMcouplings was obtained in Refs. [5]. For the example at hand the expression of the fields in terms of the seed functions is still given by Eq. (0.14), but the seed functions are no longer harmonic functions on $\mathbb{R}^{3}$; instead, they have to satisfy

$$
\vec{\partial}^{2} \mathcal{I}^{0}=0, \quad \mathfrak{D}_{i} \mathcal{I}^{l}=-\frac{1}{\sqrt{2}} \varepsilon_{i j k} \mathcal{F}_{j k}^{l},
$$

where the last equation is the Bogomol'nyi equation for the $S O(3)$ Yang-Mills-Higgs system on $\mathbb{R}^{3}$ determining the pair $\left(A_{i}^{l}, \mathcal{I}^{l}\right)$. The most famous spherically symmetric solution to the Bogomol'nyi equation is of course the 't Hooft-Polyakov monopole, which is characterized by the fact that it is completely regular. This regularity enables one to construct a globally regular, asymptotically flat supergravity solution by taking $\mathcal{I}^{0}$ to be a suitable constant [5].3.

\footnotetext{
${ }^{2}$ We shall omit the explicit expression for the Maxwell fields and refer the interested reader to Ref. [5].

${ }^{3}$ One immediate question is: Can any monopole solution to the B. equation be embedded into SUGRA theories as to give rise to a globally regular solution? The answer to this question is negative as a counterexample, to wit an $S U(4)$ monopole in the so-called $\mathbb{Q}$-magic model, can be constructed [5].
} 
Of course, the Bogomol'nyi equation admits more spherically symmetric solutions than just the 'tHP monopole [6], but not all of them have the desired properties to be used as seed solutions for constructing black hole solutions. The question of what solutions to the Bogomol'nyi equation can be used to build BH solutions was addressed in Ref. [7], and the conclusion is that apart from the 'tHP monopole, there is one family of solutions giving rise to hairy black holes with magnetic color charge $1 / g$, and an isolated solution that is similar to a colored black hole in that its asymptotic color charge vanishes.

We can study purely magnetic solutions by putting $\mathcal{I}_{\lambda}=0$, which automatically solves Eqs. (0.9) and (0.11), and then, writing $\mathcal{I}^{0}=\sqrt{2}\left(h+p r^{-1}\right)$ for convenience, the SUGRA fields for the hairy black holes are given by

$$
\vec{Z}=-\frac{\mu}{g} \frac{r H_{s}(r)}{p+h r} \vec{n}, K=(h+p / r)^{2}-\frac{\mu^{2}}{g^{2}} H_{s}^{2},
$$

where $\vec{n}$ is the outward-pointing unit vector, $\mu$ is a positive parameter measuring the vacuum expectation value of the 'Higgs' field $g \overrightarrow{\mathcal{I}}$ and the function $H_{s}(r)$ is given by

$$
H_{s}(r)=\operatorname{coth}(\mu r+s)-(\mu r)^{-1} \quad \text { with } s \geq 0 .
$$

The fundamental characteristic of the above function is that it is a monotonically increasing function on the interval $(0, \infty)$ with asymptotic values $H_{s}(r \rightarrow \infty)=1$. For $s \neq 0$, the function blows up as $r^{-1}$ around $r=0$, whereas when $s=0$ it behaves like $H_{s} \sim r$ around $r=0$; in effect, the solution with $s=0$ corresponds to the 't Hooft-Polyakov monopole.

As in the ungauged case, we can normalize the solution to asymptote to Minkowski space by putting $h=\sqrt{1+\mu^{2} g^{-2}}$, after which a small calculation shows that the mass is given by $M=h p+\mu g^{-2}$, and therefore imposes the constraint $h p>0$ in order for the mass to be positive. The globally regular solution is found by putting $s=0$ and $p=0$; in contrast, for any $s \neq 0$ we can build a black hole whenever the 'entropy is positive', which imposes

$$
S_{b h}=(p)^{2}-\frac{1}{g^{2}}>0 \text { and then we have }\left.\vec{Z}\right|_{h o r}=-\frac{1}{g p} \vec{n} .
$$

Observe that, as in the ungauged case, the near horizon solution is determined in terms of the asymptotic charges only, but that the scalars are not constant over the horizon: they form a hedgehog configuration. This is, however, hardly surprising as we are dealing with charged scalar fields on a 2 -sphere. The rôle of the parameter $s \neq 0$ is, seeing that it cannot be expressed in terms of asymptotic data such as the mass, that of a black hole hair and exemplifies the known fact that the no-hair theorems are not valid in EYM theories. The fact that the entropy doesn't depend on the hair eiher, seems fortunate when thinking about entropy calculations in string theory, but a deeper string theoretical insight into the nature of the hair is, as far as the authors are aware, lacking, but highly desirable.

The hairy black holes constructed above, seem to indicate that some kind of attractor mechanism is at work in the solutions, but as the last example shows, the story is more complicated as there are solutions whose asymptotic charges vanish: in analogy with the solutions found in EYM theories, we shall call this example a colored black hole, and is 
given by the expression

$$
\vec{Z}=-\frac{\vec{n}}{g(p+h r)\left(1+\lambda r^{2}\right)}, K=(h+p / r)^{2}-\left[g r\left(1+\lambda r^{2}\right)\right]^{-2} \quad(\lambda>0) .
$$

Normalizing the metric as above fixes $|h|=1$ and the asymptotic mass of the solution becomes $M=h p=|p|$, so that the asymptotic data are independent of the parameter $\lambda$. In order to construct a black hole, then, we need to have a non-vanishing horizon, which is readily calculated and leads to the same result as in Eq. (0.19); the resulting spacetime is best interpreted as an extreme Reissner-Nordstrom black hole surrounded by a cloud of "glue", whose fall-off is fast enough so as to not to leave an asymptotic imprint but does contribute to the horizon-geometries.

One might ask oneself about how generic these colored black holes are. At the time of writing, we know of no colored black hole in supergravity not related in one way or another to the one presented here. But we expect more, especially as the colored configurations in non-supersymmetric EYM theories are the only really non-Abelian solutions, the ones having asymptotic color charge being embedding of Abelian solutions (see e.g. Ref. [8]).

The existence of this colored black hole implies that a non-Abelian version of the attractor mechanism cannot be simply based on asymptotic charges, making the problem none the easier. In this respect it is also worth pointing out that the attractor mechanism also works for a large class of non-supersymmetric solutions, but in that case little to no analytic solutions of non-Abelian BHs are known. Apart from the attractor, the main questions for non-Abelian solutions to supergravity theories involve quantum corrections in that one would like to know if they modify/constrain the hair and whether they are compatible with the globally regular monopole solutions. On the stringy side, the fundamental question is how to build them out of fundamental constituents and to see how and if they implement hair.

\section{Acknowledgments}

This work was partially supported by the Spanish MEC grants FPA2006-00783 and FPU AP2004-2574 (MH), a MEC Juan de la Cierva scholarship (SV), the CAM grant HEPHACOS P-ESP-00346, by the EU RTN grant MRTN-CT-2004-005104, the Spanish ConsoliderIngenio 2010 program CPAN CSD2007-00042 and by the Fondo Social Europeo by means of an I3P contract(PM).

\section{References}

[1] K. Behrndt, D. Lüst and W. A. Sabra, Nucl. Phys. B 510 (1998) 264.P. Meessen, T. Ortín, Nucl. Phys. B749(2006), 291.

[2] J. Bellorín, P. Meessen, T. Ortín: Nucl. Phys. B762(2007), 229. 
[3] S. Ferrara, R. Kallosh and A. Strominger, Phys. Rev. D 52 (1995) 5412;A. Strominger, Phys. Lett. B 383 (1996) 39.

[4] A. Strominger and C. Vafa, Phys. Lett. B 379 (1996) 99.

[5] M. Hübscher, P. Meessen, T. Ortín, S. Vaulà: Phys. Rev. D78(2008), 065031; JHEP 0809(2008), 099.

[6] A.P. Protogenov: Phys. Lett. B67(1977), 62.

[7] P. Meessen, Phys. Lett. B665(2008), 388.

[8] M.S. Volkov and D.V. Gal'tsov, Phys. Rept. 319 (1999) 1 [hep-th/9810070]. 